\title{
An Unsteady Entropy Adjoint Approach for Adaptive Solution of the Shallow-Water Equations
}

\author{
Steven M. Kast ${ }^{*}$ Krzysztof J. Fidkowski $\stackrel{\text { and Philip L. Roe }}{\ddagger}$ \\ Department of Aerospace Engineering, University of Michigan, Ann Arbor, MI 48109
}

\begin{abstract}
This paper presents a novel approach to solution-based adaptation for unsteady discretizations of symmetrizable conservation laws. This approach is based on an extension of the entropy adjoint approach, which was previously introduced for steady-state simulations. Key to the approach is the interpretation of symmetrizing entropy variables as adjoint solutions for an output that states the entropy balance in the space-time computational domain. This relationship is shown for general first-order conservation laws, and it is applied to the case of the shallow water equations. Specifically, the entropy variable weighted residual is used to drive an adaptive indicator that targets regions of the space-time domain where spurious entropy generation is greatest. The error estimation and adaptation strategies are the same as those prescribed by output-based theory, with the advantage that no separate adjoint solution is required. Results for the unsteady shallow water equations in both one and two spatial dimensions show that the adaptive indicator performs better than uniform refinement and as well as or better than an unweighted residual indicator.
\end{abstract}

\section{Introduction}

Mesh-adaptive computational methods are important for producing accurate answers with efficient use of resources. They are relevant to aerospace applications, which possess a wide range of scales whose required resolution is often not known a priori. Unsteady problems can be particularly difficult in this regard, as the inclusion of time adds the possibility of both spatially and temporally localized features that require resolution. Choosing sufficient spatial and temporal resolution while maintaining computational efficiency is a difficult task. For more than very simple problems, the risk of loss of accuracy due to insufficient resolution is one that cannot be reliably managed by the user, even an expert practitioner. Instead, in such applications, robust and automated solution-based adaptive methods are critical.

Many solution-adaptive algorithms, also known as adaptive mesh refinement (AMR), rely on solution features to dictate mesh refinement.1 13 These features could be gradients, curvatures, or any other directly computable solution characteristics. Such indicators are inexpensive computationally, but they provide no guarantees on the quality of the solution. Even in steady-state simulations, adapting on solution features can lead to poor performance, especially for predicting engineering outputs 45

*Research Assistant

${ }^{\dagger}$ Assistant Professor, AIAA Member

‡Professor, AIAA Fellow 
More robust are output-based adaptive algorithms, which produce meshes specifically suited for the prediction of a scalar output of interest. These methods also return error estimates that improve robustness of the output calculation. While the topic of output error estimation and mesh adaptation has been studied in depth for steady problems, ${ }^{15}$ unsteady problems have received considerably less attention.12 14 One reason for this is that while in the steady case the cost of the adjoint solution is generally no more than that of the original forward problem (implementation fixed costs aside), the cost rises in the unsteady case, especially in terms of storage for nonlinear problems.

For steady-state, a compromise between heuristic and output-based adaptive indicators was introduced as the "entropy adjoint" approach 15 This method appealed to the observation that the entropy state variables act as an adjoint solution for an output that expresses an entropy balance statement in the computational domain. Adaptation driven by the entropy adjoint approach was demonstrated to produce "all-around" good solutions that competed with engineering output adjoint solutions in terms of output accuracy, even though the generation and transport of entropy in the domain is generally not of direct engineering interest.

In the present work, we extend the entropy adjoint approach to unsteady problems. In this case, the output becomes an entropy balance statement that accounts for the entropy inflow and outflow through all boundaries of the space-time domain. That is, the accounting includes the entropy present in the initial condition, the entropy present at the final time, and the net entropy that leaves the spatial domain during the course of the simulation.

We derive the entropy adjoint output expression for general, unsteady first-order conservation laws, and we present adaptive numerical results for one instructive system: the shallow water equations. These nonlinear equations govern the depth (height) and velocity of fluid in free surface flows. They are hyperbolic, exhibiting steep gradients or discontinuities, and hence are similar in character to flows of aerodynamic interest. The system has no separate thermodynamic entropy; instead, it is the total energy that gives rise to "entropy variables" that symmetrize the equations. We stress that it is the symmetrizing property of the entropy variables, not necessarily a thermodynamic connection, that is the foundation for the entropy-adjoint approach.

The error estimate from the unsteady entropy adjoint approach is localized in the space-time discretization to provide information on regions in the space-time computational domain that are important for the prediction of the entropy balance output. This indicator can be used to drive a general adaptive method that refines/coarsens the spatial and temporal discretizations as appropriate. In the present results, we use this indicator to drive combinations of temporal and spatial refinement in both one-dimensional and two-dimensional unsteady cases.

The outline for the remainder of this paper is as follows. Section II derives the connection between entropy variables and unsteady adjoint solutions, for first order conservation laws. Section III presents the shallow water equations in conservative form, the finite volume and finite element discretizations, and the symmetrizing entropy variables. Next, Section IV outlines the error estimation and mesh adaptation algorithms. Finally, results are given in Section $\mathrm{V}$ for two test cases: a one-dimensional problem solved via the finite volume method and adapted dynamically in space, and a two-dimensional problem solved via the discontinuous Galerkin method and adapted simultaneously in both space and time. 


\section{The Unsteady Entropy Adjoint Connection}

Consider a system of partial differential equations arising from an unsteady conservation law,

$$
\mathbf{r}(\mathbf{u})=\partial_{t} \mathbf{u}+\partial_{i} \mathcal{F}_{i}=\mathbf{0}
$$

where $\mathbf{u}(\mathbf{x}, t) \in \mathcal{V}$ is the vector of conservative state variables, $\mathcal{F}_{i}$ is a general flux that may consist of convective and diffusive components, and $i$ in the implied summation indexes the spatial dimension. The computational domain is assumed to be a tensor product between a spatial domain $\Omega$ and a temporal domain $T$, where

$$
\mathbf{x} \in \Omega, \quad t \in T=\left[t_{0}, t_{f}\right]
$$

We will denote by $\partial \Omega$ the boundary of $\Omega$, and by $\partial T$ the boundary of $T$ (i.e. $t_{0}$ and $t_{f}$ ). The theory is not tied to a tensor-product space-time domain (e.g. moving geometries can be incorporated), but the following exposition is less cumbersome in the tensor-product case.

\section{A. The Unsteady Adjoint Equation and Boundary Conditions}

In a continuous setting, an adjoint is a Green's function that relates residual source perturbations in the original equation to an output of interest. Equivalently, it is a Lagrange multiplier on the residual when used in constrained optimization. The latter definition allows for a rigorous discussion of the adjoint equations and boundary conditions, which arise in the discussion of the entropy variables in the next sub-section.

Given a scalar output $J(\mathbf{u})$, we define a Lagrangian as

$$
\mathcal{L}=J(\mathbf{u})-\int_{T} \int_{\Omega} \psi^{T} \mathbf{r}(\mathbf{u}) d \Omega d t
$$

where the Lagrange multiplier, $\boldsymbol{\psi} \in \mathcal{V}$, is the adjoint solution] $][16$ Enforcing stationarity of $\mathcal{L}$ with respect to permissible variations in the state yields the adjoint equation,

$$
J^{\prime}[\mathbf{u}] \delta \mathbf{u}-\int_{T} \int_{\Omega} \boldsymbol{\psi}^{T} \mathbf{r}^{\prime}[\mathbf{u}] \delta \mathbf{u} d \Omega d t=0, \quad \forall \delta \mathbf{u} \in \mathcal{V}^{\text {perm }},
$$

where $\mathcal{V}^{\text {perm }}$ denotes the space of permissible state variations, that is, those allowed by the boundary conditions. The primes above denote Fréchét linearization with respect to the arguments in square brackets. Using the conservation form in Eqn. 1, Eqn. 3 can be integrated by parts to yield an interior differential equation and boundary conditions for the adjoint,

$$
\begin{aligned}
J^{\prime}[\mathbf{u}] \delta \mathbf{u}= & \int_{T} \int_{\Omega} \boldsymbol{\psi}^{T} \mathbf{r}^{\prime}[\mathbf{u}] \delta \mathbf{u} d \Omega d t \\
= & \int_{T} \int_{\Omega} \boldsymbol{\psi}^{T}\left[\partial_{t} \delta \mathbf{u}+\partial_{i}\left(\mathcal{F}_{i}^{\prime}[\mathbf{u}] \delta \mathbf{u}\right)\right] d \Omega d t \\
= & -\int_{T} \int_{\Omega}\left[\partial_{t} \boldsymbol{\psi}^{T}+\partial_{i} \boldsymbol{\psi}^{T} \mathcal{F}_{i}^{\prime}[\mathbf{u}]\right] \delta \mathbf{u} d \Omega d t \\
& +\int_{T} \int_{\partial \Omega} \boldsymbol{\psi}^{T} \mathcal{F}_{i}^{\prime}[\mathbf{u}] \delta \mathbf{u} n_{i} d S d t+\left[\int_{\Omega} \boldsymbol{\psi}^{T} \delta \mathbf{u} d \Omega\right]_{t_{0}}^{t_{f}},
\end{aligned}
$$


where $d S$ is the differential area element on $\partial \Omega$ and $n_{i}$ is the $i^{\text {th }}$ component of the outward pointing spatial normal on $\partial \Omega$.

The standard next step is to assert that the first term in the final right-hand-side of Eqn. 4 is the adjoint equation on the interior of the space-time domain, so that

$$
\partial_{t} \boldsymbol{\psi}+\left(\mathcal{F}_{i}^{\prime}[\mathbf{u}]\right)^{T} \partial_{i} \boldsymbol{\psi}=0, \quad \forall \delta \mathbf{u} \in \mathcal{V}^{\text {perm }},
$$

while the second and third terms are associated with the boundary and initial conditions, respectively. Specifically, on pure Dirichlet inflow boundary conditions, the permissible primal variation is $\delta \mathbf{u}=0$, and hence $\boldsymbol{\psi}$ is not constrained, while at pure outflow boundary conditions, $\delta \mathbf{u}$ is not constrained, and hence $\boldsymbol{\psi}$ is constrained. Similarly, initial conditions only constrain $\delta \mathbf{u}=0$ at $t=t_{0}$, so that $\boldsymbol{\psi}$ is not constrained at $t=t_{0}$ but fully constrained at $t=t_{f}$. This duality in constraints represents the "reversal" in initial and boundary conditions when considering the adjoint problem.

However, the constraints on $\psi$ at the outflow boundary conditions and $t=t_{f}$ do not necessarily mean that information has to be specified there. If the last two terms in Eqn. 4 have counterparts in $J^{\prime}[\mathbf{u}](\delta \mathbf{u})$, the adjoint constraints will be satisfied. This is the case for the entropy variables, as discussed in the next sub-section.

We note that in the "interior adjoint equation", Eqn. 5, a negative sign appears on both the spatial and temporal derivative terms following the respective integration by parts, so that there is no relative sign change between these two terms compared to the primal equation. In addition, Eqn. 5, does not constitute the complete adjoint equation when $J$ contains contributions from the interior of the space-time domain.

\section{B. Entropy Variables as Unsteady Adjoints}

At present we restrict our attention to first order conservation laws, for which we write $\mathcal{F}_{i}^{\prime}[\mathbf{u}]=\mathbf{A}_{i}$. The conservative variables then satisfy the following quasi-linear system of equations.

$$
\partial_{t} \mathbf{u}+\mathbf{A}_{i} \partial_{i} \mathbf{u}=0
$$

We assume the existence of a scalar entropy conservation law,

$$
U_{t}+\partial_{i} F_{i}=0
$$

where $U$ is an entropy function (e.g. proportional to the physical entropy for the Euler equations) and $F_{i}(\mathbf{u})$ is the entropy flux associated with $U(\mathbf{u})$. The entropy conservation law holds only if the compatibility relation $U_{\mathbf{u}} \mathbf{A}_{i}=\left(F_{i}\right)_{\mathbf{u}}$ is satisfied. For a convex entropy function $U$, the set of corresponding entropy variables is defined by $\mathbf{v} \equiv U_{\mathbf{u}}^{T}$. The entropy variables symmetrize the conservation laws in the sense that $2[17$

- the transformation Jacobian matrix, $\mathbf{u}_{\mathbf{v}}$, is symmetric, positive definite,

- $\mathbf{A}_{i} \mathbf{u}_{\mathbf{v}}$ is symmetric. 
Using these symmetry properties, the quasi-linear conservation law in Eqn. 6 can be manipulated as follows:

$$
\begin{aligned}
0 & =\partial_{t} \mathbf{u}+\mathbf{A}_{i} \partial_{i} \mathbf{u} \\
& =\mathbf{u}_{\mathbf{v}} \partial_{t} \mathbf{v}+\mathbf{A}_{i} \mathbf{u}_{\mathbf{v}} \partial_{i} \mathbf{v} \\
& =\mathbf{u}_{\mathbf{v}} \partial_{t} \mathbf{v}+\left(\mathbf{A}_{i} \mathbf{u}_{\mathbf{v}}\right)^{T} \partial_{i} \mathbf{v} \\
& =\mathbf{u}_{\mathbf{v}} \partial_{t} \mathbf{v}+\mathbf{u}_{\mathbf{v}}^{T} \mathbf{A}_{i}^{T} \partial_{i} \mathbf{v} \\
& =\mathbf{u}_{\mathbf{v}} \partial_{t} \mathbf{v}+\mathbf{u}_{\mathbf{v}} \mathbf{A}_{i}^{T} \partial_{i} \mathbf{v}
\end{aligned}
$$

Since $\mathbf{u}_{\mathbf{v}}$, is symmetric, positive definite, we multiply the above equation by $\mathbf{u}_{\mathbf{v}}^{-1}$ to obtain

$$
\partial_{t} \mathbf{v}+\mathbf{A}_{i}^{T} \partial_{i} \mathbf{v}=0
$$

A comparison of this equation to Eqn. 5, with $\mathbf{A}_{i}=\mathcal{F}_{i}^{\prime}[\mathbf{u}]$, suggests that the entropy variables satisfy an adjoint equation for an output that has no contributions from the interior of the spacetime domain. To verify the validity of this assertion and to determine the associated output, we substitute the entropy variables in place of $\boldsymbol{\psi}$ in the output linearization, Eqn. 4. The first term drops out, leaving

$$
J^{\prime}[\mathbf{u}] \delta \mathbf{u}=\int_{T} \int_{\partial \Omega} \mathbf{v}^{T} \mathbf{A}_{i} \delta \mathbf{u} n_{i} d S d t+\left[\int_{\Omega} \mathbf{v}^{T} \delta \mathbf{u} d \Omega\right]_{t_{0}}^{t_{f}} .
$$

Now, $\mathbf{v}^{T} \mathbf{A}_{i} \delta \mathbf{u}=\left(F_{i}\right)_{\mathbf{u}} \delta \mathbf{u}=\delta F_{i}$ and $\mathbf{v}^{T} \delta \mathbf{u}=U_{\mathbf{u}} \delta \mathbf{u}=\delta U$, so that

$$
J=\int_{T} \int_{\partial \Omega} F_{i} n_{i}+\left[\int_{\Omega} U\right]_{t_{0}}^{t_{f}}+\text { constant. }
$$

Since initial conditions prescribe $\mathbf{u}$ at $t=t_{0}$, and hence constrain $\delta \mathbf{u}$ there, $\int_{\partial T}$ just reduces to evaluation at $t=t_{f}$ up to the arbitrary constant in Eqn. 8. Thus, the output associated with the entropy variables is an entropy balance statement for the computational domain. The first term in Eqn. 8 is the net outflow of the entropy function $U$ through the spatial domain boundary, integrated over time. The second term is the net increase of the entropy function integrated over the spatial domain $\Omega$, between $t_{0}$ and $t_{f}$. For the exact solution, these two terms should balance and $J$ should be zero. However, $J$ need not be zero in a numerical simulation on a discretized space-time mesh because most conservative schemes do not conserve entropy.

\section{Discretization and Solution}

In the present study we consider the shallow water equations, which fall into the category of first-order conservation laws, Eqn. 1. In two spatial dimensions, the state and flux vectors are

$$
\mathbf{u}=\left[\begin{array}{c}
h \\
u h \\
v h
\end{array}\right], \quad \mathcal{F}_{1}=\left[\begin{array}{c}
u h \\
u^{2} h+g h^{2} / 2 \\
u v h
\end{array}\right], \quad \mathcal{F}_{2}=\left[\begin{array}{c}
v h \\
u v h \\
v^{2} h+g h^{2} / 2
\end{array}\right]
$$

where $h$ is the fluid depth (height), $u$ and $v$ are the depth-averaged velocities in the $x_{1}$ and $x_{2}$ directions, respectively, and $g$ is the gravitational acceleration. 
The shallow water equations can be symmetrized by expressing them in terms of an appropriate set of entropy variables, $\frac{18}{18}$

$$
\mathbf{v}=\left[\begin{array}{c}
g h-\left(u^{2}+v^{2}\right) / 2 \\
u \\
v
\end{array}\right]
$$

These entropy variables correspond to the following entropy function and fluxes:

$$
U=\frac{g h^{2}+h\left(u^{2}+v^{2}\right)}{2}, \quad F_{1}=u\left(g h^{2}+\frac{h\left(u^{2}+v^{2}\right)}{2}\right), \quad F_{2}=v\left(g h^{2}+\frac{h\left(u^{2}+v^{2}\right)}{2}\right) .
$$

We note that in this system, no thermodynamic entropy is defined, and instead the entropy function $U$ is the sum of the potential energy and the kinetic energy in the fluid column. 18

In the present work, we consider the shallow water equations in both one and two dimensions. The discretizations and solution methods for these cases are described in the following sections.

\section{A. One-Dimensional Finite Volume Discretization}

In one dimension, only $\mathcal{F}_{1}$ is present, and the variable $v$ is set to zero everywhere. The onedimensional shallow water equations are discretized in space using a first-order finite volume method, and in time using an implicit backward Euler scheme. We denote by $\mathbf{U}_{H}^{k} \in \mathbb{R}^{s N_{H}^{k}}$ the vector of unknowns on time slab $k \in 1 \ldots K$, for which $t \in\left(t_{k-1}, t_{k}\right]$. $N_{H}^{k}$ is the number of cells on time slab $k$, and $s=2$ is the number of partial differential equations in the system. A temporally constant solution on each time slab is consistent with an $r=0$ DG in time interpretation (see next section) of backward Euler. ${ }^{19}$ We consider dynamic spatial refinement of the grid, so that $N_{H}^{k}$ is variable in time.

The discretized form of the one-dimensional conservation law reads

$$
\overline{\mathbf{R}}_{H}^{k}\left(\mathbf{U}_{H}^{k}, \mathbf{U}_{H}^{k-1}\right) \equiv \mathbf{M}_{H}^{k}\left(\mathbf{U}_{H}^{k}-\mathbf{U}_{H}^{k-1}\right)+\Delta t \mathbf{R}_{H}\left(\mathbf{U}_{H}^{k}\right)=\mathbf{0}
$$

where $\overline{\mathbf{R}}_{H}^{k}$ is the unsteady residual associated with time slab $k, \mathbf{M}_{H}^{k} \in \mathbb{R}^{s N_{H}^{k} \times s N_{H}^{k}}$ is the spatial mass matrix that includes the cell lengths, and $\mathbf{R}_{H}\left(\mathbf{U}_{H}^{k}\right)$ is the spatial residual vector that incorporates the details of the spatial discretization, which is performed using the Roe flux function, as presented in. 20 Note that $\overline{\mathbf{R}}_{H}^{k}$ depends on the state at time slab $k$ and $k-1$. Since the spatial grid may change between time slabs, the solution from slab $k-1$ is projected onto the grid associated with slab $k$ before the $\mathbf{U}_{H}^{k-1}$ term in $\overline{\mathbf{R}}_{H}^{k}$ is calculated. For example, for a cell on time slab $k$ with left and right boundaries at locations $x_{i}$ and $x_{i+1}$, respectively, there may be several cells on time slab $k-1$ within the same spatial range. In this case, $\mathbf{U}_{H}^{k-1}$ is taken to be the weighted average of the cells on the slab $k-1$ grid between $x_{i}$ and $x_{i+1}$.

\section{B. Two-Dimensional Discontinuous Galerkin Discretization}

In two dimensions, a discontinuous Galerkin (DG) finite element discretization is used in both space and time. Details on the discretization can be found in.21 Briefly, for order $r$ approximation in time, the $r+1$ discrete residual vectors, indexed by $m \in 1 \ldots r+1$, on one time slab $k$ are

$$
\overline{\mathbf{R}}_{H}^{k, m}\left(\mathbf{U}_{H}^{k, n}, \mathbf{U}_{H}^{k-1, n}\right) \equiv a_{r}^{m, n} \mathbf{M}_{H} \mathbf{U}_{H}^{k, n}-\varphi_{H}^{m}\left(t_{k-1}\right) \mathbf{M}_{H} \mathbf{U}_{H}^{k-1, r+1}+\int_{t_{k-1}}^{t_{k}} \varphi_{H}^{m}(t) \mathbf{R}_{H}\left(\mathbf{U}_{H}^{k}(t)\right) d t=\mathbf{0}
$$


In this expression $n \in 1 \ldots r+1$ is a time node index within a time slab, and the discrete vectors $\mathbf{U}_{H}^{k, n}, \overline{\mathbf{R}}_{H}^{k, m}, \mathbf{R}_{H}^{k}$ are of size $N_{H}$, which is the number of spatial degrees of freedom (assumed constant in time). Summation is implied on the repeated index $n$. In the spatial discretization, the Roe linearization ${ }^{22}$ is used for the inviscid flux and the second form of Bassi and Rebay ${ }^{23}$ is used for the viscous flux. In addition, the $\varphi_{H}^{m}(t)$ are temporal basis functions, $\mathbf{M}_{H}$ is the spatial mass matrix, $\mathbf{U}_{H}^{k-1, r+1}$ is the state at the end of the previous time slab, and $a_{r}^{m, n}=-\int_{t_{k-1}}^{t_{k}} \varphi_{H}^{n} \frac{d \varphi_{H}^{m}}{d t} d t+$ $\varphi_{H}^{n}\left(t_{k}\right) \varphi_{H}^{m}\left(t_{k}\right)$. Note the dependence of the residual on the states at time slab $k$ and $k-1$.

Due to the first and last terms in Eqn. 13, all degrees of freedom within a time slab are coupled together. Each time slab is also coupled to its predecessor through the solution at the end of the previous slab. We solve this system using an iterative method based on an approximate linearization, 19.21 which only requires solutions of systems that have the same size and (block) sparsity as the spatial Jacobian.

The two-dimensional results include output-based error estimation and mesh adaptation, which are based on the solution of a discrete adjoint equation. The $r+1$ adjoint residuals on time slab $k$ are obtained by linearizing and transposing the residuals in Eqn. 13 , resulting in

$$
\overline{\mathbf{R}}_{H}^{k, k, n}\left(\boldsymbol{\Psi}_{H}^{k, m}, \boldsymbol{\Psi}_{H}^{k+1, m}\right) \equiv\left(\frac{\partial \overline{\mathbf{R}}_{H}^{k, m}}{\partial \mathbf{U}_{H}^{k, n}}\right)^{T} \boldsymbol{\Psi}_{H}^{k, m}+\left(\frac{\partial \overline{\mathbf{R}}_{H}^{k+1, m}}{\partial \mathbf{U}_{H}^{k, n}}\right)^{T} \boldsymbol{\Psi}_{H}^{k+1, m}-\left(\frac{\partial J_{H}}{\partial \mathbf{U}_{H}^{k, n}}\right)^{T}=\mathbf{0}
$$

where $J_{H}$ is the output of interest, and $\boldsymbol{\Psi}_{H}^{k, m}$ is the discrete adjoint vector on time node $m$ of time slab $k$. These equations are solved using the same approximate factorization technique used for the forward solution, in a time-reversed simulation.

\section{Error Estimation and Mesh Adaptation}

We estimate the numerical error in a scalar output, $J$, computed from the discrete solution, using the adjoint-weighted residual method. Details on the theory are reviewed in ${ }^{11}$ In this work, we estimate the output perturbation relative to a finer space that is obtained by uniformly refining the temporal discretization and uniformly refining (1D) or incrementing the order (2D) of the spatial discretization.

We denote by $\mathbf{U}_{h}^{H, k, n}$ the coarse solution injected into the fine space, where $n \in 1 \ldots r+1$ indexes the time nodes per slab ( $r=0$ in the Backward Euler case). There is no loss of approximation in this injection as the coarse space is a subset of the fine space. Weighting the fine-space unsteady residual computed with $\mathbf{U}_{h}^{H, k, n}$ by the unsteady adjoint on the fine space yields the output error estimate,

$$
\delta J \approx \sum_{k=1}^{K} \sum_{m=1}^{r+1}\left(\delta \mathbf{\Psi}_{h}^{k, m}\right)^{T} \overline{\mathbf{R}}_{h}^{k, m}\left(\mathbf{U}_{h}^{H, k, n}, \mathbf{U}_{h}^{H, k-1, n}\right)
$$

where $\delta \mathbf{\Psi}_{h}^{k, m}=\boldsymbol{\Psi}_{h}^{k, m}-\boldsymbol{\Psi}_{h}^{H, k, m}, \mathbf{\Psi}_{h}^{k, m}$ is the discrete vector representing the fine-space adjoint, and $\boldsymbol{\Psi}_{h}^{H, k, m}$ is the injection of the coarse adjoint into the fine space. Since the entropy variables are unsteady adjoints, using them in place of $\boldsymbol{\Psi}$ in Eqn. 15 yields an estimate of the error in the entropy balance output defined in Eqn. 8 .

The fine-space adjoint/entropy variables $\Psi_{h}^{k, m}$ can be approximated using reconstructions or approximate solutions. However, in the present work, we solve for $\Psi_{h}^{k, m}$ to machine zero residual 
on the fine space to eliminate unquantified error sources. We do not expect much deterioration of the adaptive method when adjoint approximations are employed.

The output error calculation in Eqn. 15 can be recast as a sum over space-time cells in the coarse mesh,

$$
\delta J \approx \sum_{k=1}^{K} \sum_{e=1}^{N_{H}} \varepsilon_{k, e},
$$

where the error contribution of a coarse space-time element in spatial cell $e$ and time slab $k$ is

$$
\varepsilon_{k, e}=\sum_{m \in \operatorname{dof}_{h}\left(\mathcal{I}_{k}^{H}\right)} \sum_{i \in \operatorname{dof}_{h}\left(\mathcal{T}_{e}^{H}\right)}\left(\Psi_{h, i}^{k, m}\right)^{T} \overline{\mathbf{R}}_{h, i}^{k, m}\left(\mathbf{U}_{h}^{H, k, n}, \mathbf{U}_{h}^{H, k-1, n}\right),
$$

where $\operatorname{dof}_{h}\left(\mathcal{I}_{k}^{H}\right)$ is the set of fine time slab indices corresponding to the coarse time slab index $k$, and $\operatorname{dof}_{h}\left(\mathcal{T}_{e}^{H}\right)$ is the set of fine cell indices corresponding to the coarse spatial cell index $e$. Note that both $\boldsymbol{\Psi}_{h, i}^{k, m}$ and $\overline{\mathbf{R}}_{h, i}^{k, m}$ are vectors in $\mathbb{R}^{s} . \overline{\mathbf{R}}_{h, i}^{k, m}$ is the unsteady residual associated with spatial degree of freedom $i$ and temporal degree of freedom $m$ on time slab $k$. The error indicator for a space-time cell is obtained by taking the absolute value of the elemental contribution,

$$
\text { error indicator }=\epsilon_{k, e}=\left|\varepsilon_{k, e}\right| \text {. }
$$

This indicator identifies the space-time cells most responsible for the error in the targeted output, $J$. In general, the adaptive strategy is to reduce the output error by refining those cells responsible for the largest amount of error in the output. However, in the following results, we use a more conservative error indicator in which absolute values are placed around the error contributions associated with each conservation equation prior to summation.

The above error indicator ascribes a certain amount of error to each space-time cell but does not provide information about whether this error is associated with the spatial or temporal discretization. To determine whether the cell should be refined in space or time, a measure of space-time anisotropy is required. In the present work, we use an anisotropy measure based on solution jumps, so that the amount of error associated with each timeslab and spatial element is

$$
\begin{aligned}
& \text { temporal error on time slab } k=\epsilon_{k}=\sum_{e=1}^{N_{H}} \epsilon_{k, e} \beta_{k, e}^{\mathrm{time}} \\
& \text { spatial error on element } \mathrm{k}, \mathrm{e}=\epsilon_{k, e}^{\text {space }}=\epsilon_{k, e} \beta_{k, e}^{\text {space }}
\end{aligned}
$$

where $\beta_{k, e}^{\text {time }}$ is defined as the ratio of the average temporal (across time slabs) jump to the sum of the temporal and spatial (across spatial cells) jumps, and

$$
\beta_{k, e}^{\text {space }}=1-\beta_{k, e}^{\text {time }} .
$$

Finally, to determine which elements to refine, the errors associated with each timeslab and spatial cell are divided by the number of new degrees of freedom that would be generated upon refinement. In $1 \mathrm{D}$, a fixed fraction strategy is then used to refine a fraction $f^{\text {adapt }}$ of the spatial elements and/or time slabs with the highest ratio of error to additional degrees of freedom. In $2 \mathrm{D}$, a fixed growth strategy is used in which the number of elements/slabs refined is based on a

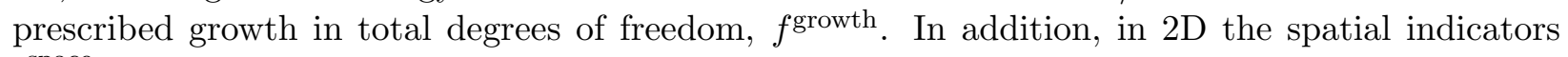
$\epsilon_{k, e}^{\text {space }}$ are summed over slabs $k$ to drive a spatially-static adaptive strategy.

$$
8 \text { of } 20
$$




\section{Results}

This section presents a computational verification of the equivalence between the entropy variables and the adjoint corresponding to the output in Eqn. 8, as well as adaptive results for oneand two-dimensional unsteady cases solved via the finite volume and the discontinuous Galerkin method, respectively. Performance of the entropy adaptation is measured in terms of accuracy of domain and boundary integral outputs relative to uniform and residual-based adaptation.

\section{A. Verification of the Unsteady Entropy-Adjoint Connection}

To verify that the entropy variables do in fact serve as an adjoint solution to the output in Eqn. 8 , comparisons between the entropy variables and the actual adjoint are presented for sample oneand two-dimensional unsteady problems solved via DG with approximation order $p$ in space and $r$ in time.

In the first case, a sinusoidal perturbation in fluid height in one spatial dimension is allowed to evolve from rest under the influence of gravity. The fluid height contours for this case are shown in Figure 1. From the contours, we see that the initial perturbation splits in half as it falls, forming a set of waves moving in opposite directions, which then interact with the walls and each other as they propagate. In two dimensions, an initial Gaussian perturbation in fluid height, situated in the center of a square domain as shown in Figure 1, is allowed to evolve in time.

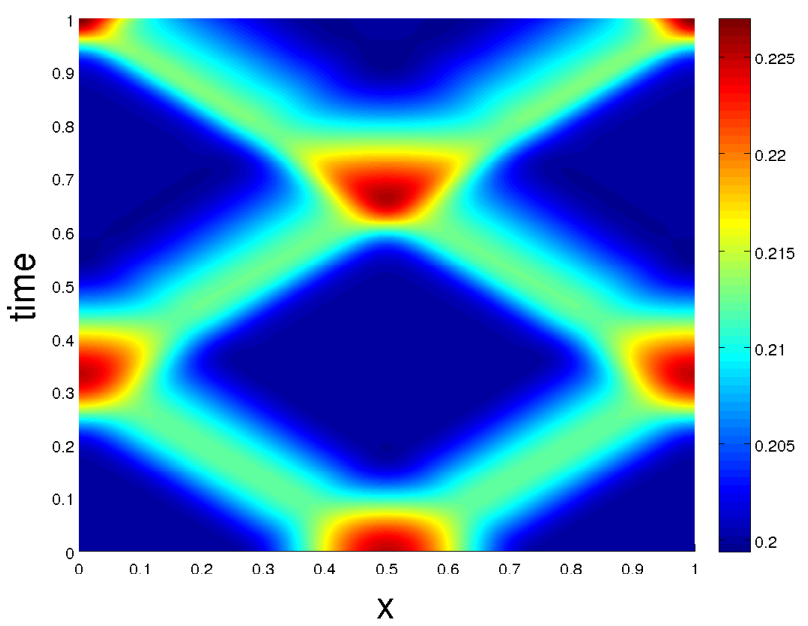

(a) 1D space-time fluid height contours

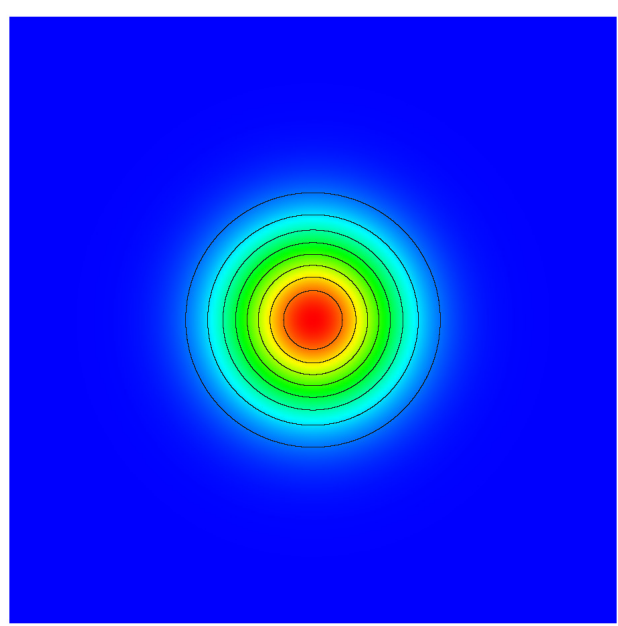

(b) 2D initial fluid height: contours from 0.2 (blue) to 0.225 (red)

Figure 1. Test cases used for verification of the entropy adjoint connection in one and two dimensions.

Figures 2 and 3 compare contours of the first entropy variable (corresponding to the mass conservation equation), computed by a direct transformation of the primal solution, to the adjoint variable solved separately, in a discrete fashion by marching backwards in time via Eqn. 14 for the output in Eqn. 8. The qualitative similarity between the two can be readily seen. We note that the quantities are not identical as they are different finite dimensional approximations to the same continuous adjoint solution: the entropy variables.

As a quantitative measure of similarity, the $L_{2}$ norm of the error between entropy variables and 


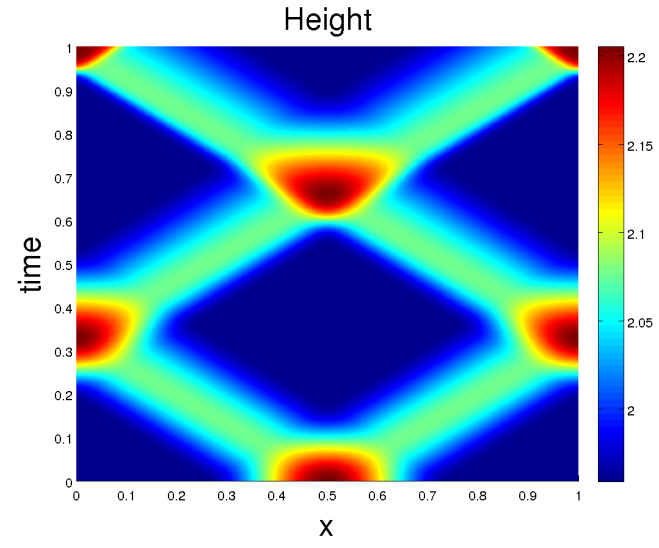

(a) Entropy variable (first component)

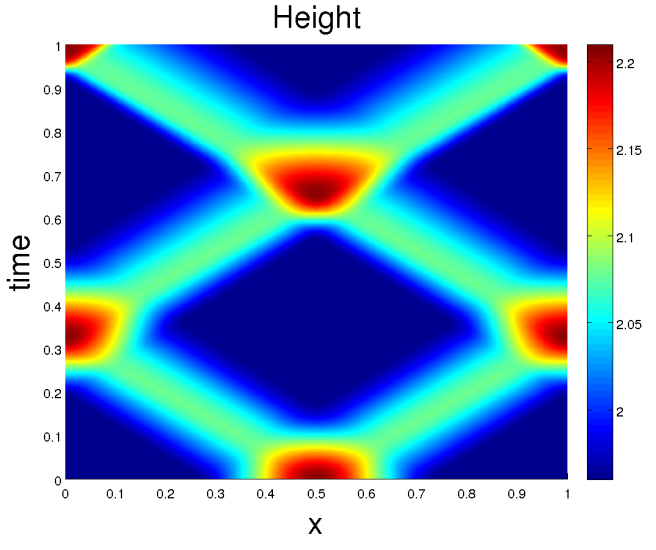

(b) Output adjoint (first component)

Figure 2. Qualitative illustration of equivalence between entropy variables and the unsteady adjoint corresponding to the output in Eqn. 8 for a one-dimensional problem.

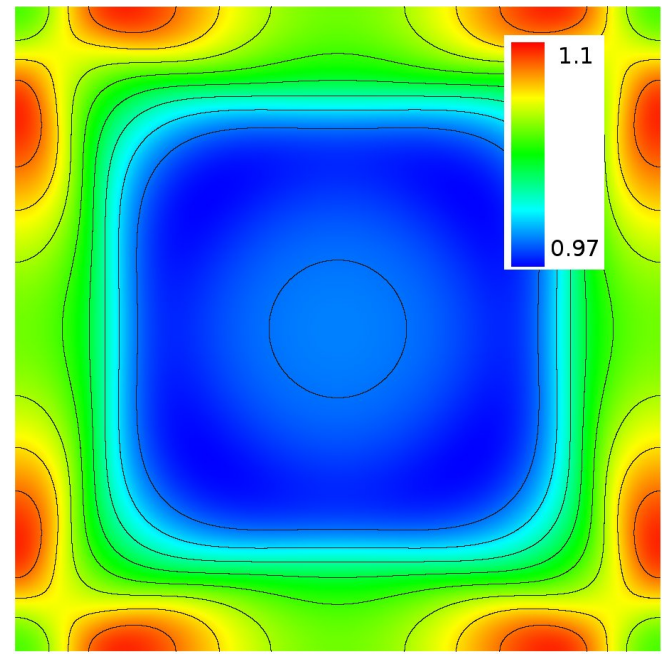

(a) Entropy variable (first component)

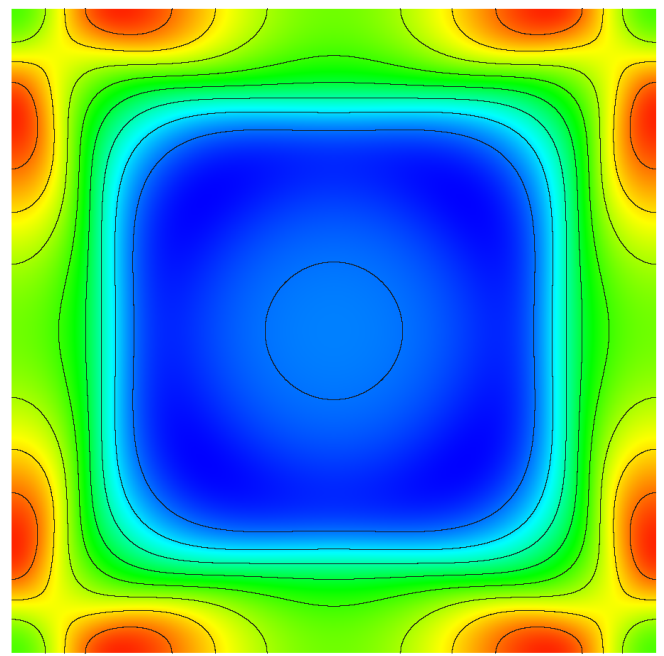

(b) Output adjoint (first component)

Figure 3. Qualitative illustration of equivalence between entropy variables and the unsteady adjoint corresponding to the output in Eqn. 8 for a two-dimensional problem. Spatial contours shown at one time, the middle of the temporal domain. 
the adjoint,

$$
\text { entropy variable adjoint error }=\left(\int_{T} \int_{\Omega}\left\|\boldsymbol{\psi}_{h}-\mathbf{v}_{h}\right\|^{2} d \Omega d t\right)^{1 / 2},
$$

is calculated for both cases, and its convergence with uniform space and time refinement is shown in Figure 4 for different choices of approximation orders $p$ and $r$. Although asymptotic convergence rates, which are expected to be $\min (p+1,2 r+1)$, are not yet achieved in the refinements shown, the error does decrease upon mesh refinement, so that the entropy variables approach the actual adjoint solution.

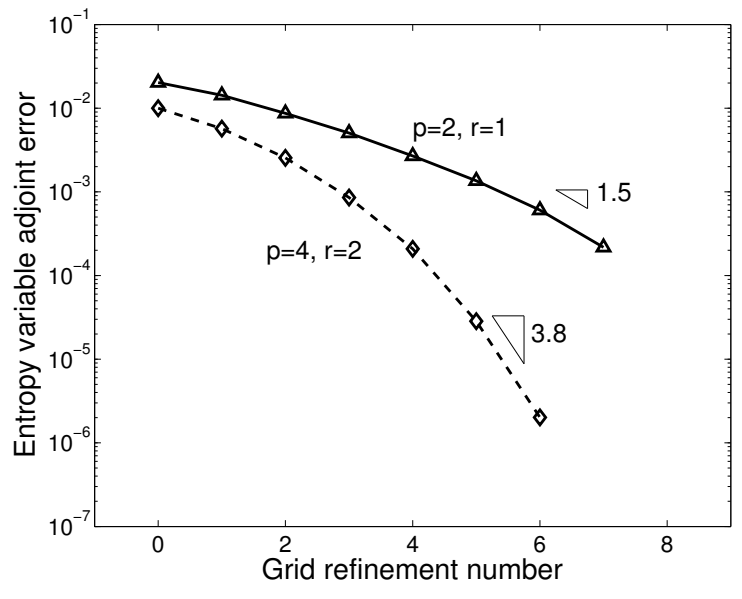

(a) One spatial dimension

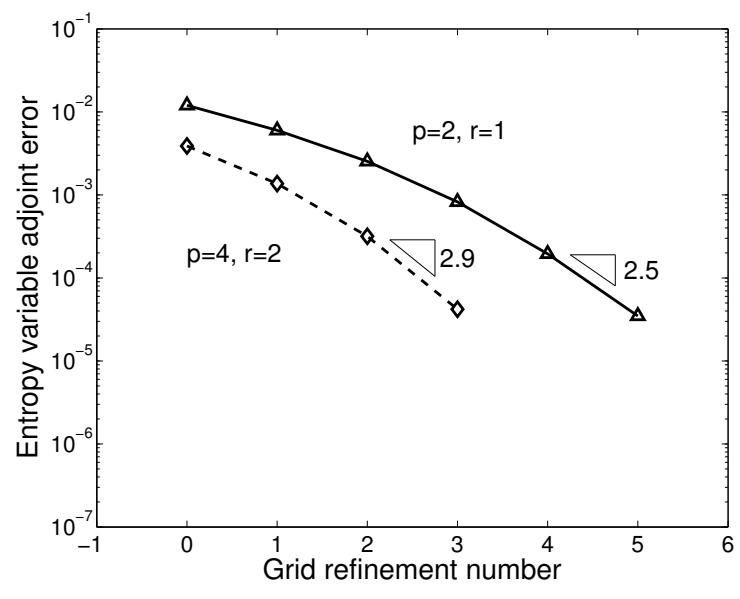

(b) Two spatial dimensions

Figure 4. $L_{2}$ error between entropy variables and the adjoint variables corresponding to the output in Eqn. 8, Convergence shown for uniform refinement in both space and time, using order $p$ for spatial approximation, and order $r$ for temporal approximation in a discontinuous Galerkin spacetime formulation.

\section{B. One-Dimensional Sinusoidal Wave}

In this section, we present adaptive results for a one-dimensional unsteady case solved via the first-order finite volume method. The initial condition for this problem $(t=0$ in Figure 5 ) consists of a sinusoidal perturbation in fluid with a velocity that is zero where the height is constant and otherwise positive and proportional to the magnitude of the perturbation in height. The fluid is allowed to evolve for one second in time under the influence of gravity $\left(9.81 \mathrm{~m} / \mathrm{s}^{2}\right)$, and its behavior is shown in Figures 5 and 6 .

From the contours and solution profiles, we see that a depression in the fluid forms behind the initial positive perturbation, and the two mirror each other as they propagate throughout the space-time domain, reflecting off the boundaries and colliding briefly before reaching the opposite ends of the domain by the end of the simulation. The $u h$, or momentum, contour shows that while the depression propagates initially to the left, the actual fluid in the depression travels to the right, replacing the fluid that leaves with the rightward-moving wave and thereby satisfying conservation. During the collision near $t=0.7$, the interaction between the perturbation and depression produces a cancellation effect, and the height of the perturbation is reduced slightly, as expected. 


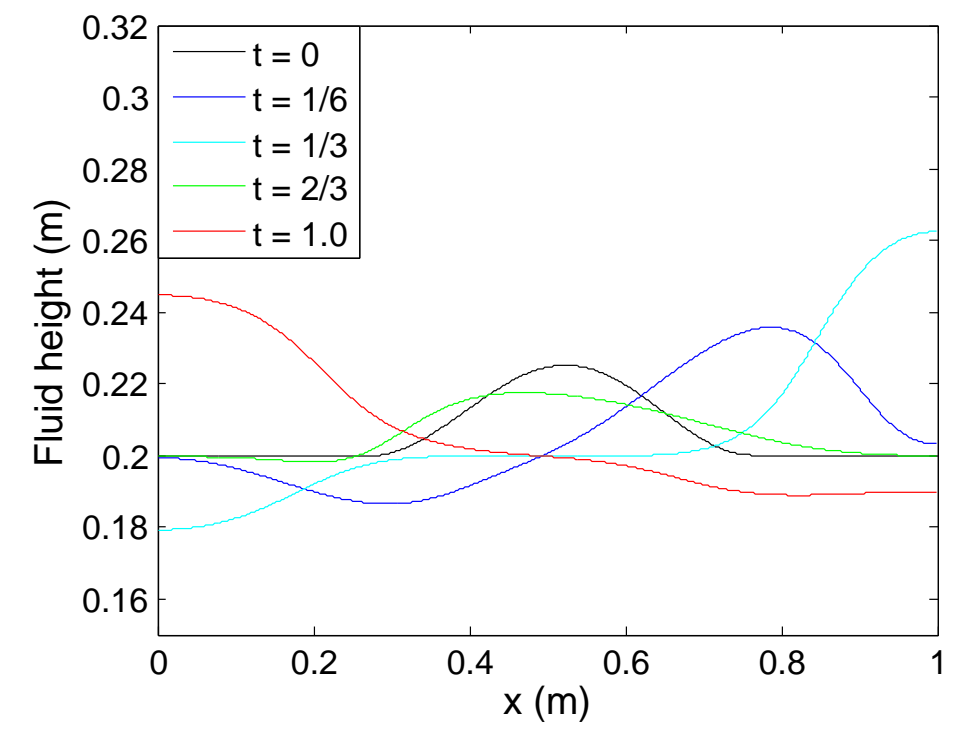

Figure 5. One-dimensional sinusoidal wave: fluid height snapshots at various times.

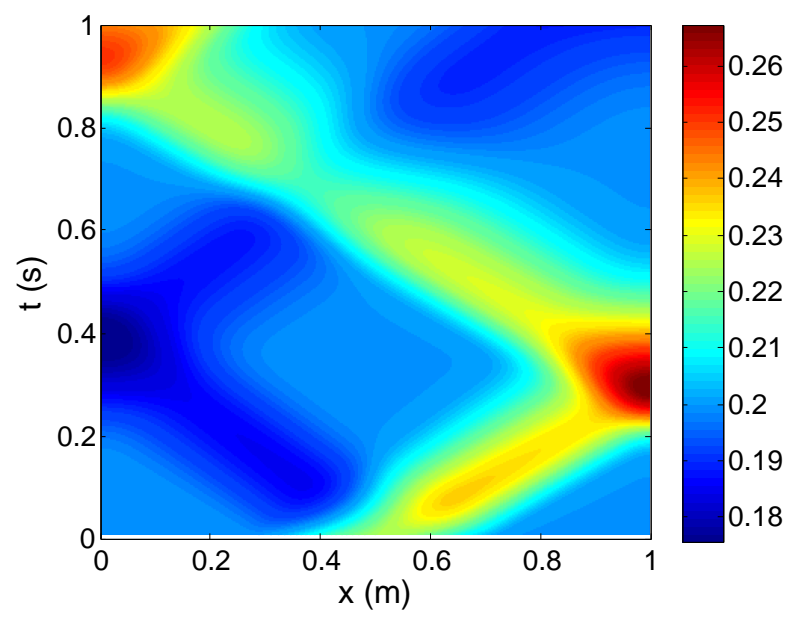

(a) Fluid height, $h$

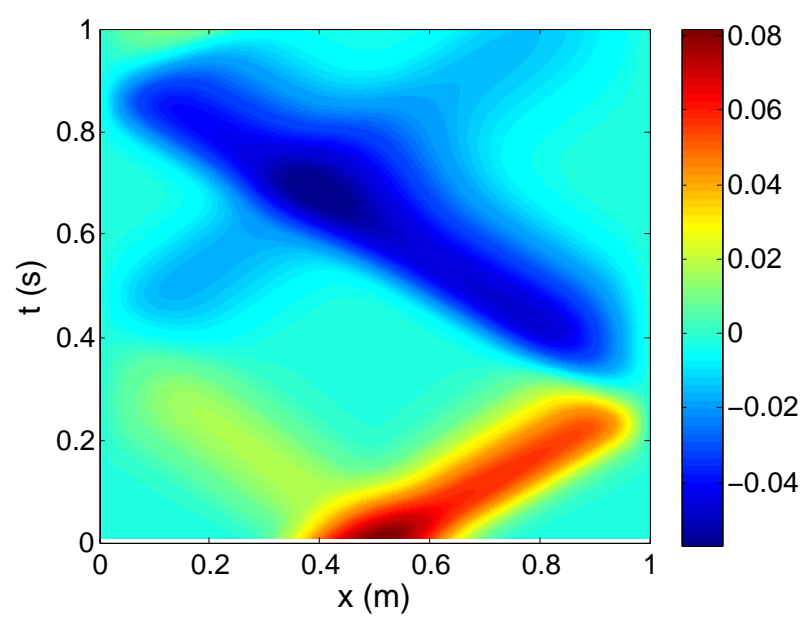

(b) Fluid momentum, uh

Figure 6. One-dimensional sinusoidal wave: space-time state contours of the solution on a fine mesh. 


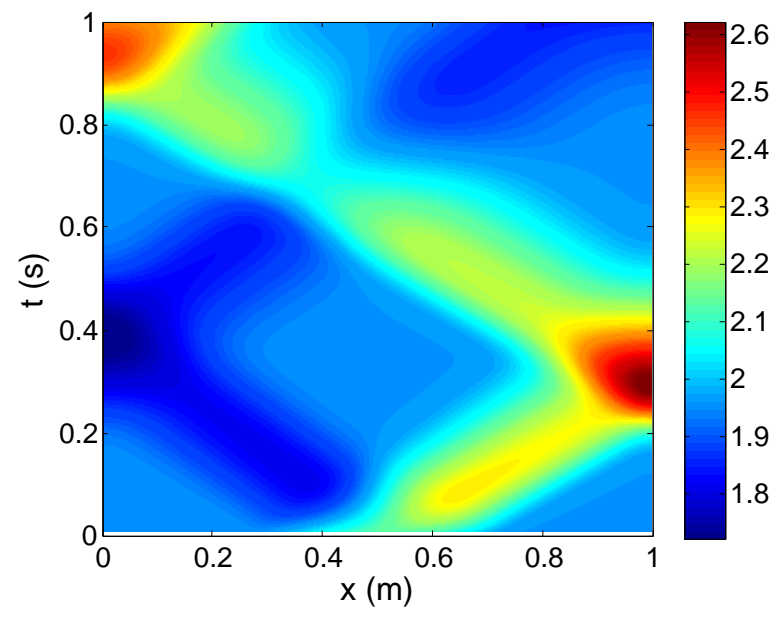

(a) First entropy variable

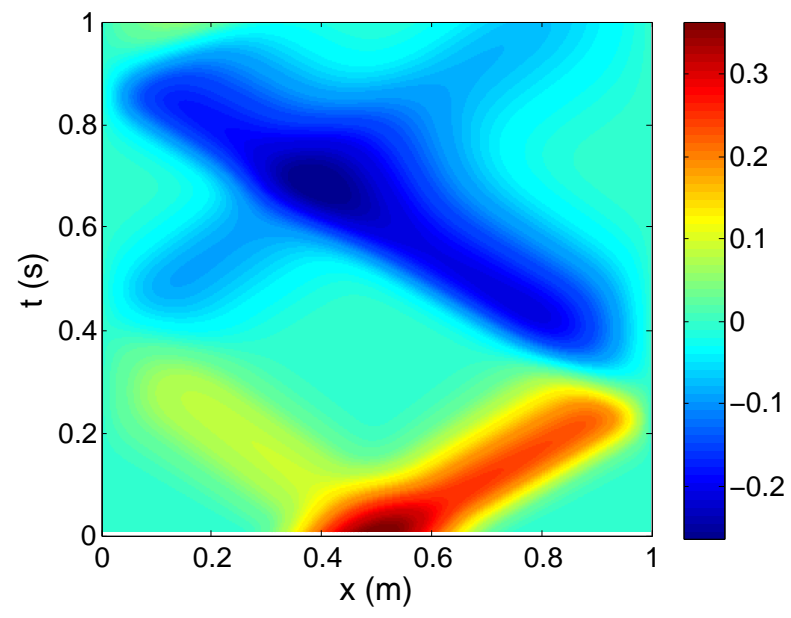

(b) Second entropy variable

Figure 7. One-dimensional sinusoidal wave: space-time entropy variable contours of the solution on a fine mesh. Note that due to the relative magnitude of $h$ and $u h$ in this problem, the entropy variable contours show a strong correlation to the state contours.

The adaptation strategy for this problem consists of dynamic refinement of the spatial grid in time, with the temporal grid (consisting of 150 uniformly-spaced nodes) remaining constant for all adaptations. A constant temporal grid is not a requirement, but we found that for this 1D problem, using solution jumps to weight the residual is not a particularly effective way to apportion the error. Instead, we present only spatial adaptation for this case, and show combined spatial and temporal adaptation in the two-dimensional problem presented in the following section.

The outputs of interest in this simulation were chosen to be the square of the conserved variables integrated over the space-time domain,

$$
\begin{gathered}
J_{1}=\int_{T} \int_{\Omega} h^{2} d x d t, \\
J_{2}=\int_{T} \int_{\Omega}(u h)^{2} d x d t .
\end{gathered}
$$

As integrals over the entire space-time domain, $J_{1}$ and $J_{2}$ are taken to reflect the convergence of the entire solution. The convergence of these outputs is shown for three different spatial adaptation strategies in Figure 8. Degrees of freedom, which is the total number of space-time elements in the grid, is chosen as a surrogate for the computational cost.

The first adaptation strategy is simply uniform spatial refinement, achieved via bisection of each spatial cell. Next is a "heuristic" adaptive indicator, which consists of the fine-space residual weighted by the solution-jump anisotropy measure. The final indicator, and that of primary interest in this paper, is based on the entropy adjoint, which is the fine space residual weighted by the entropy variables. Figure 8 shows that the entropy adjoint and residual-based strategies display markedly similar convergence rates, both performing better than uniform refinement and requiring approximately 40 percent fewer degrees of freedom to reach convergence. This gain relative to uniform refinement is consistent with expectations for adaptation in only one dimension. 


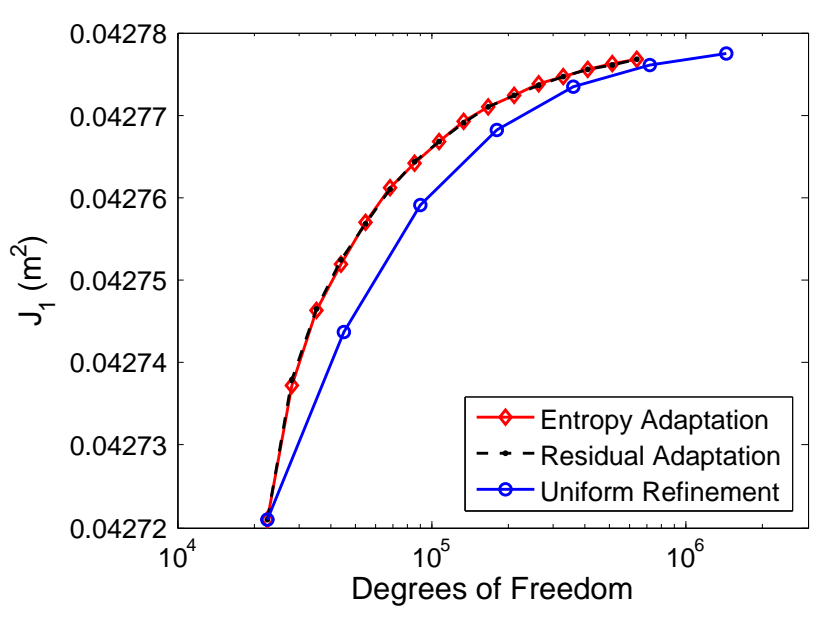

(a) $h^{2}$ integrated over space-time domain

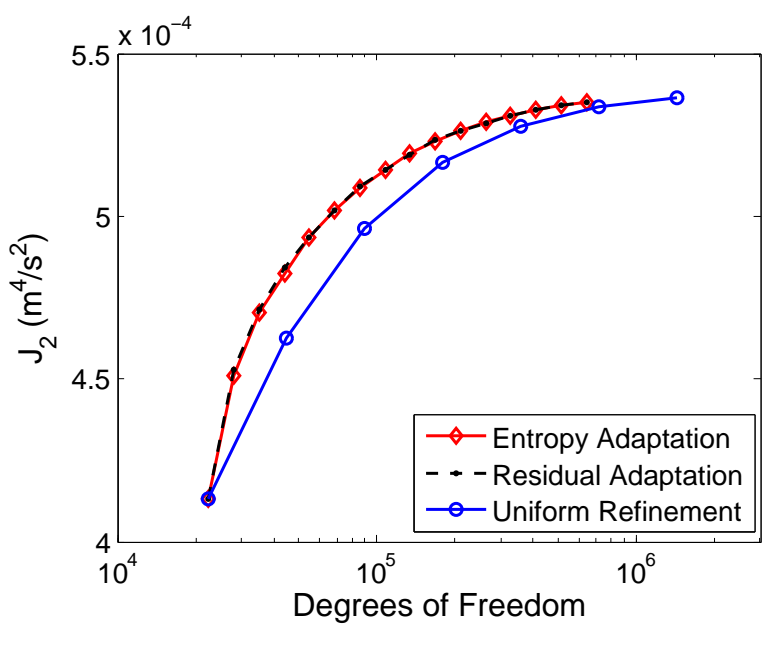

(b) $(u h)^{2}$ integrated over space-time domain

Figure 8. One-dimensional sinusoidal wave: convergence of outputs which are the integral of $h^{2}$ and $(u h)^{2}$ over the space-time domain for three adaptation strategies. ( $f^{\text {adapt }}=.25$ for adaptive methods).

The adapted meshes for the entropy-adjoint and residual indicators are shown in Figure 9, For clarity, the temporal nodes (which would appear as horizontal lines in the grid) have been omitted, and only the spatial cell boundaries are shown.

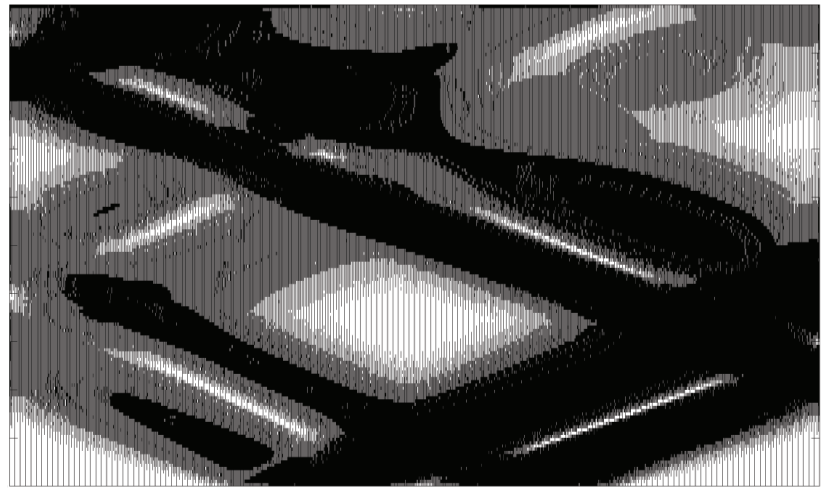

(a) Entropy grid after 12 adaptations

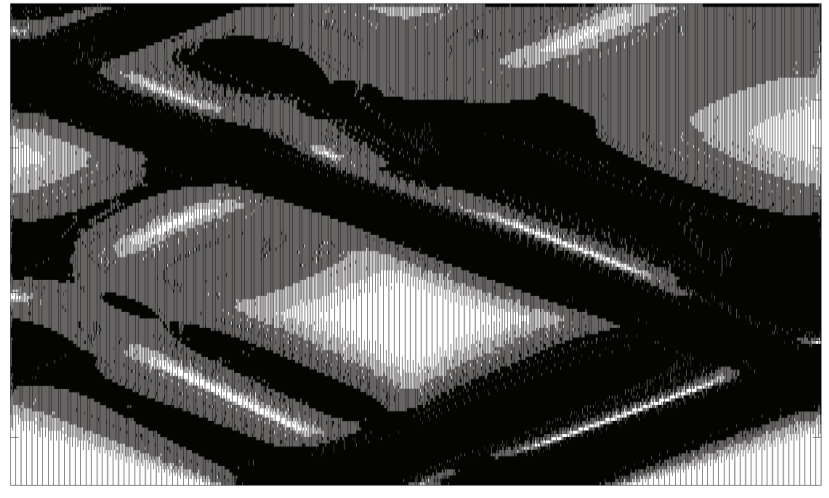

(b) Residual grid after 12 adaptations

Figure 9. One-dimensional sinusoidal wave: grids for entropy-adjoint and residual adaptation methods.

We see that the entropy and residual indicator target similar regions, refining around the perturbation and depression as they propagate throughout the domain and leaving the areas of stagnant fluid unrefined. The residual indicator appears to target the depression slightly more than the entropy indicator, while the entropy indicator refines more around the perturbation after it has collided with the depression. As expected, the entropy indicator generally appears to target the regions where the entropy variables are large in magnitude, as shown in Figure 7. 


\section{Two-Dimensional Depth Disturbance}

In this problem a cylinder geometry interacts with disturbances that emanate from a two-dimensional Gaussian depth distribution at $t=0$. The Gaussian depth distribution, shown in Figure 10 a, is centered 7 cylinder radii away from the cylinder origin. It has an amplitude $30 \%$ above the nominal depth of one cylinder radius, and a variance of $1 / 2$ cylinder radii squared. The fluid velocity is zero everywhere at $t=0$.

The simulation extends to a time $t_{f}=12$, measured in units based on the cylinder radius and gravitational acceleration (both set to unity for convenience). The fluid depth at the half-way time, $t=6$, is shown in Figure 10p. Note that at this time the cylinder is in the midst of interacting with the radial wave propagating from the disturbance center.

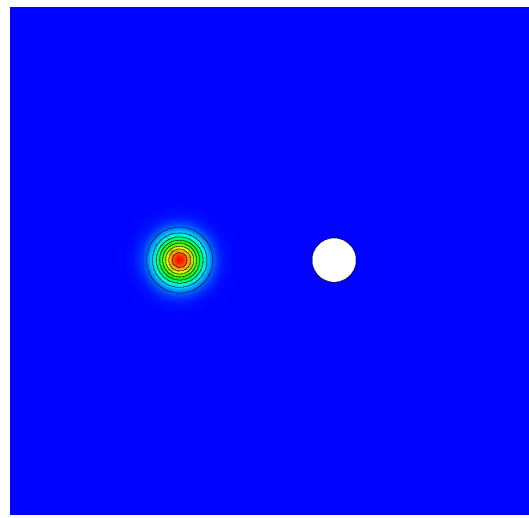

(a) Fluid height at $t=0$ : contours from 1.0 (blue) to 1.3 (red)

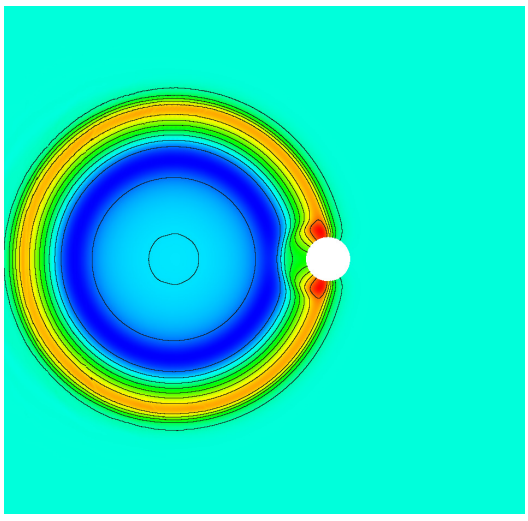

(b) Fluid height at $t=t_{f} / 2$ : contours from 0.98 (blue) to 1.05 (red)

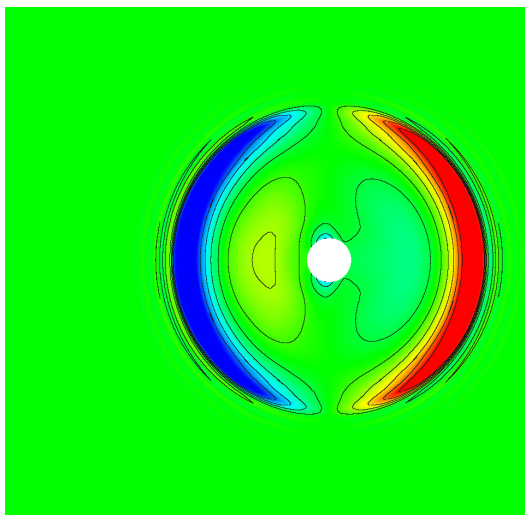

(c) Fluid height adjoint at $t=t_{f} / 2$ : contours from -0.2 (blue) to 0.2 (red)

Figure 10. Two-dimensional depth disturbance simulation: initial condition and primal/adjoint solutions half-way through the simulation.

This problem is discretized using a discontinuous Galerkin finite element method in both space and time. The solution approximation order is $p$ in space and $r$ in time, on prismatic (tensor product basis) space-time elements.

The output of interest in this simulation is the time integral of the horizontal force (integrated momentum flux) on the cylinder. Adaptive simulations are performed from an initial mesh that is coarse in both space and time. Specifically, the coarse space-time mesh consists of 16 time slabs and the spatial mesh illustrated in Figure 12 , with $p=1$ spatial approximation order and $r=1$ temporal approximation order.

Uniform refinement and three adaptive indicators are compared in their ability to efficiently predict the output: entropy-adjoint adaptation; adaptation driven by the adjoint of the output of interest; and adaptation driven by the unweighted residual. The temporal adaptation consists of time slab bisection at a fixed $r=1$. In space, two static adaptive strategies are considered: static $h$-refinement, in which the quadrilateral elements are refined in a hanging-node fashion, and static $p$-refinement, in which the approximation orders on individual elements are increased. We note that "static" in this context refers to the spatial mesh resolution remaining fixed in time.

Figure 11 shows the convergence of the output under the various adaptive indicators, for both $h$ and $p$ spatial refinement. Error bars obtained by the adjoint-weighted residual are included with the output adjoint results. The "actual" value of the output is obtained by Richardson extrapolation of 


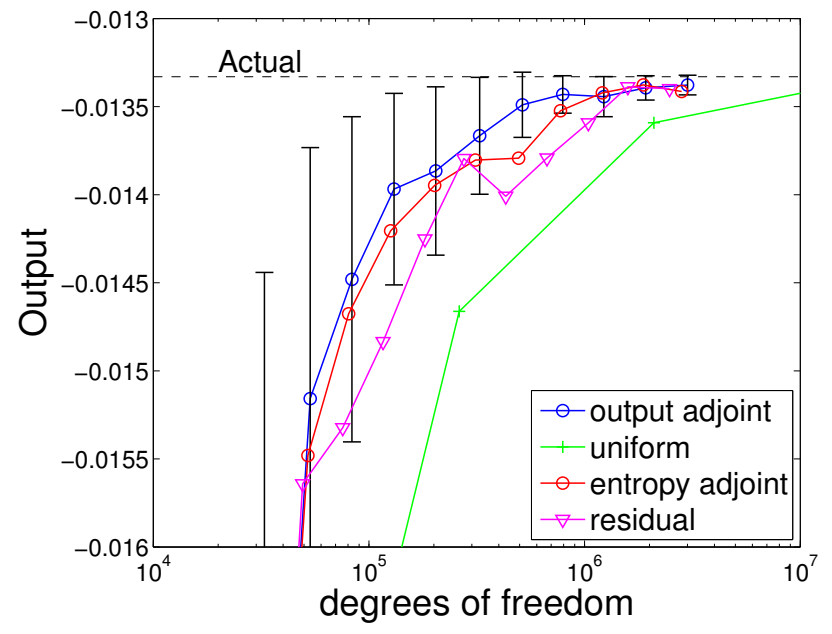

(a) $h$-refinement

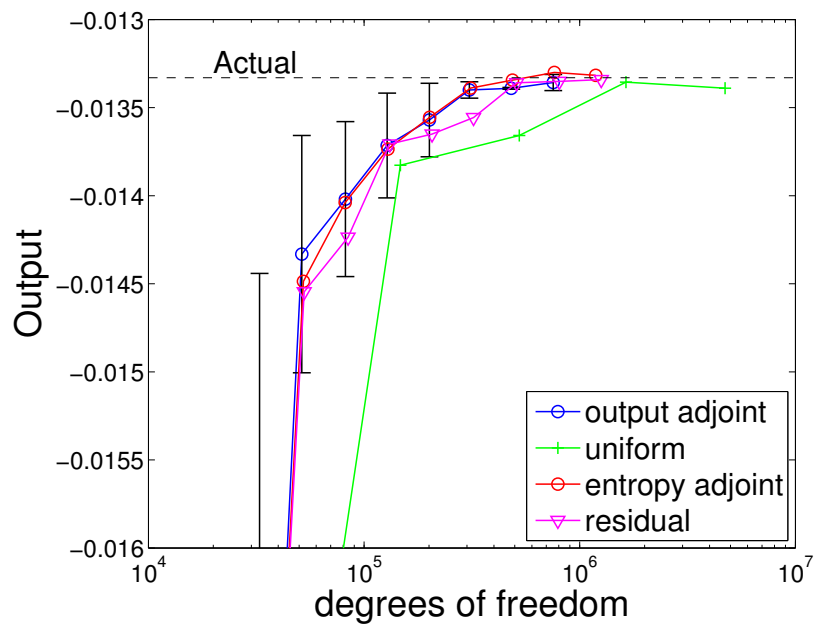

(b) $p$-refinement

Figure 11. Two-dimensional depth disturbance: convergence of an output which is the time integral of the $x$-direction force on the immersed cylinder geometry.

the uniform $h$-refinement results, using two additional uniform refinements beyond those shown. As expected, uniform refinement converges the slowest in terms of degrees of freedom for a given output accuracy. In contrast, the indicator based on the output adjoint yields the most rapid convergence rate. We note that the error bars are quite accurate after a few initial adaptive iterations. The next most rapid convergence is observed, in general, from the entropy-adjoint indicator, followed by the residual indicator. The differences among the indicators are apparent primarily in the $h$-refinement strategy; under $p$-refinement, the spread in performance is much smaller, likely due to the efficiency with which high $p$ resolves the spatially-smooth solution.

Figures 12 and 13 show the spatial meshes for various indicators at the fifth adaptive iteration of $h$-refinement and $p$-refinement, respectively. We see that in the $h$-refinement cases, the residual and entropy indicators target similar regions, focusing on the location of the initial Gaussian disturbance and the left-hand side of the domain. The output indicator, on the other hand, ignores the portion of the wave propagating away from the cylinder, and instead clusters its refinement closely around the cylinder to accurately predict the incident force. The spatial regions targeted for $p$-refinement are similar to those targeted in the $h$-refinement cases.

Figure 14 shows the temporal meshes for the $h$ and $p$ refinements at the sixth adaptation. Note that the temporal domain is more refined in the $p$-refinement cases, since increasing approximation order is more efficient at reducing error than $h$-refinement for this smooth problem, so that more degrees of freedom can be used to adapt temporally. Overall, all indicators refine the temporal grids in a relatively uniform manner. The output-based indicator has a slight preference for the middle of the temporal domain, the entropy adjoint indicator for the initial times, and the residual for the end of the simulation. Presumably, the residual adaptation skips the initial times because, since the disturbance is localized, the average residual over the whole domain at those times is small. Instead, it refines temporally after the wave has spread out, since then the wave covers a larger spatial region and therefore increases the average residual. The entropy indicator seems to target the initial times since, although the initial disturbance is localized, the entropy variables are large and likely not well-approximated in this region. Finally, the output indicator's preference for 
the middle of the temporal domain can be explained by the fact that these are the times of closest interaction between the cylinder and the wave, and therefore are most important to the accurate prediction of the incident force.

\section{Conclusions}

This paper presents an unsteady extension of the entropy adjoint approach to adaptive mesh refinement. For first-order conservation laws and tensor-product space-time domains, the output associated with using the entropy variables as adjoint solutions is shown to be an entropy balance statement that accounts for the entropy inflow and outflow through all boundaries of the space-time domain. We expect this result to hold for more general domains and for symmetrizable second-order conservation laws, and such investigations are the subject of ongoing work.

The adaptive indicator obtained from the entropy-variable weighted residual provides information on the areas of the domain most responsible for numerical error in the entropy balance output. These are understood as regions in space-time where spurious entropy is created. Adapting on this indicator then targets one general measure of numerical error.

The present work deals with the shallow water equations, and the results provided show the advantage of using the entropy adjoint approach in unsteady mesh adaptation. Although output adjoints are expected to provide the most robust strategy for targeting specific outputs, adapting on the entropy balance output via the entropy adjoints appears to produce accurate "general-purpose" solutions without the cost of a separate unsteady adjoint solution. Future work will apply these ideas to the unsteady Euler and Compressible Navier-Stokes equations.

\section{References}

\footnotetext{
${ }^{1}$ Baker, T. J., "Mesh adaptation strategies for problems in fluid dynamics," Finite Elements in Analysis and Design, Vol. 25, 1997, pp. 243-273.

${ }^{2}$ Barth, T. J., "Numerical methods for gasdynamic systems on unstructured meshes," An Introduction to Recent Developments in Theory and Numerics for Conservation Laws, Proceedings of the International School on Theory and Numerics for Conservation Laws, Berlin, Lecture Notes in Computational Science and Engineering, edited by D. Kröner, M. Ohlberger, and C. Rhode, Springer-Verlag, 1999.

${ }^{3}$ Warren, G. P., Anderson, W. K., Thomas, J. L., and Krist, S. L., "Grid convergence for adaptive methods," AIAA Paper 1991-1592, 1991.

${ }^{4}$ Zhang, X. D., Vallet, M.-G., Dompierre, J., Labbe, P., Pelletier, D., Trepanier, J.-Y., Camarero, R., Lassaline, J. V., Manzano, L. M., and Zingg, D. W., "Mesh adaptation using different error indicators for the Euler equations," AIAA Paper 2001-2549, 2001.

${ }^{5}$ Venditti, D. A. and Darmofal, D. L., "Anisotropic grid adaptation for functional outputs: application to two-dimensional viscous flows," Journal of Computational Physics, Vol. 187, No. 1, 2003, pp. $22-46$.

${ }^{6}$ Pierce, N. A. and Giles, M. B., "Adjoint recovery of superconvergent functionals from PDE approximations," SIAM Review, Vol. 42, No. 2, 2000, pp. 247-264.

${ }^{7}$ Becker, R. and Rannacher, R., "An optimal control approach to a posteriori error estimation in finite element methods," Acta Numerica, edited by A. Iserles, Cambridge University Press, 2001, pp. 1-102.

${ }^{8}$ Hartmann, R. and Houston, P., "Adaptive discontinuous Galerkin finite element methods for the compressible Euler equations," Journal of Computational Physics, Vol. 183, No. 2, 2002, pp. 508-532.

${ }^{9}$ Sen, S., Veroy, K., Huynh, D., Deparis, S., Nguyen, N., and Patera, A., "“Natural norm" a posteriori error estimators for reduced basis approximations," Journal of Computational Physics, Vol. 217, 2006, pp. 37-62.

${ }^{10}$ Nemec, M. and Aftosmis, M. J., "Error estimation and adpative refinement for embedded-boundary Cartesian meshes," AIAA Paper 2007-4187, 2007.

${ }^{11}$ Fidkowski, K. J. and Darmofal, D. L., "Review of output-based error estimation and mesh adaptation in computational fluid dynamics," American Institute of Aeronautics and Astronautics Journal, Vol. 49, No. 4, 2011, pp. 673-694.

${ }^{12}$ Mani, K. and Mavriplis, D. J., "Discrete adjoint based time-step adaptation and error reduction in unsteady
} 


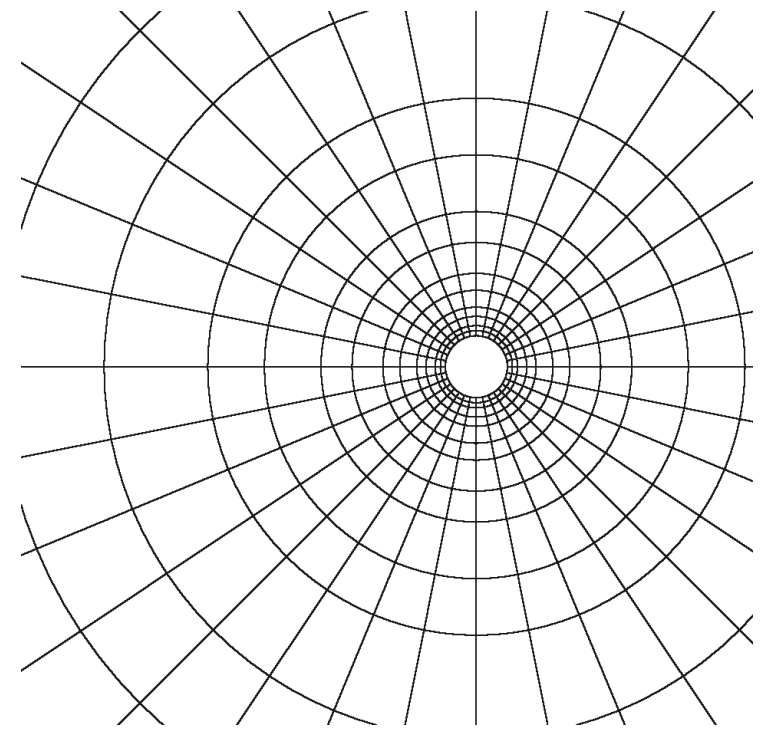

(a) Initial mesh

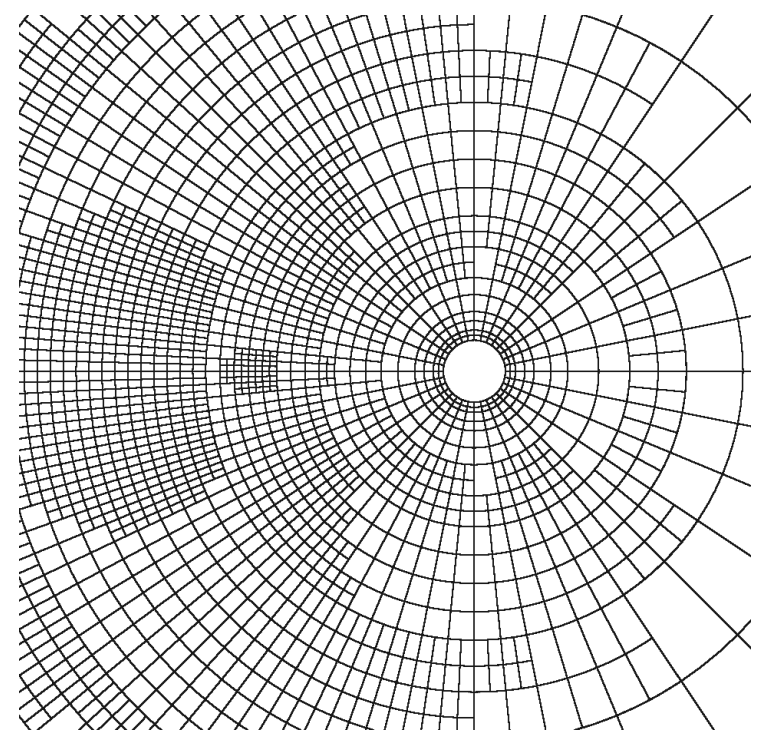

(c) Entropy adjoint

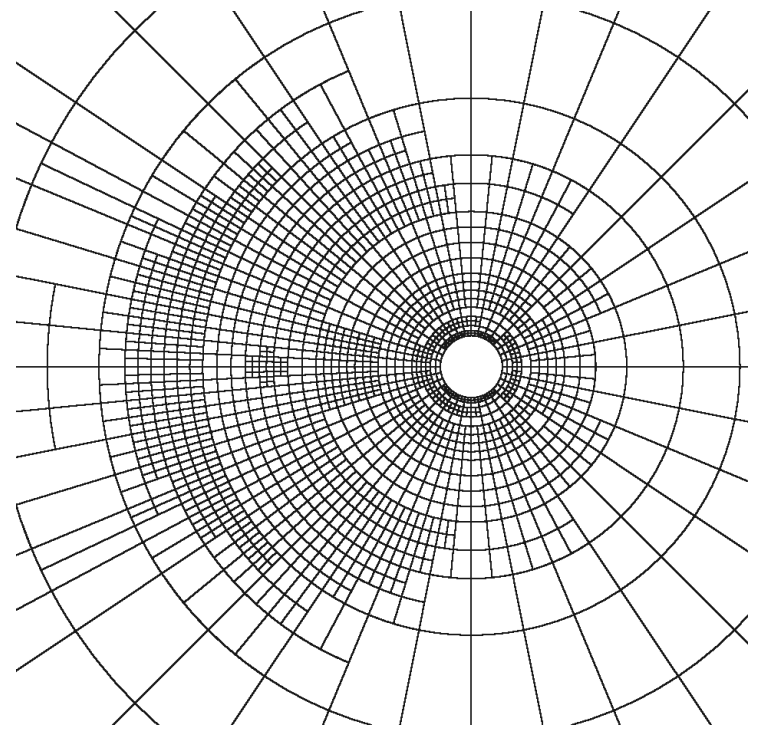

(b) Output adjoint

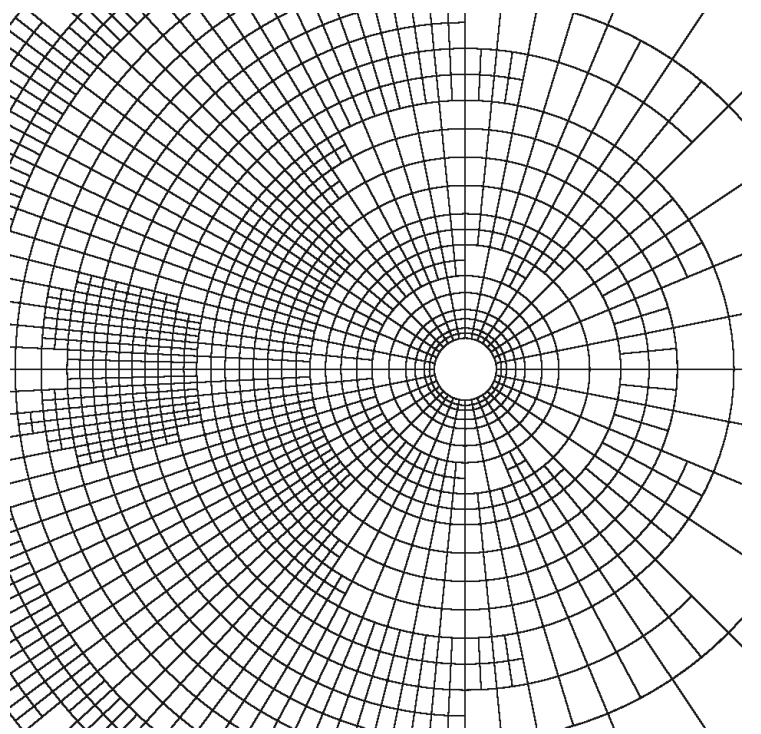

(d) Residual

Figure 12. Two-dimensional depth disturbance: $h$-refinement meshes at the fifth adaptation iteration for various indicators. 


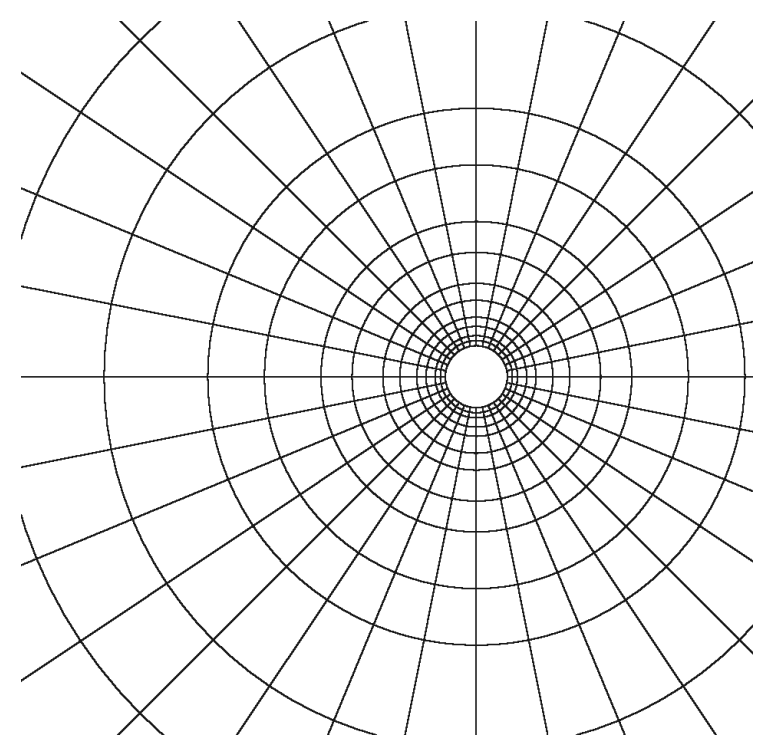

(a) Initial mesh

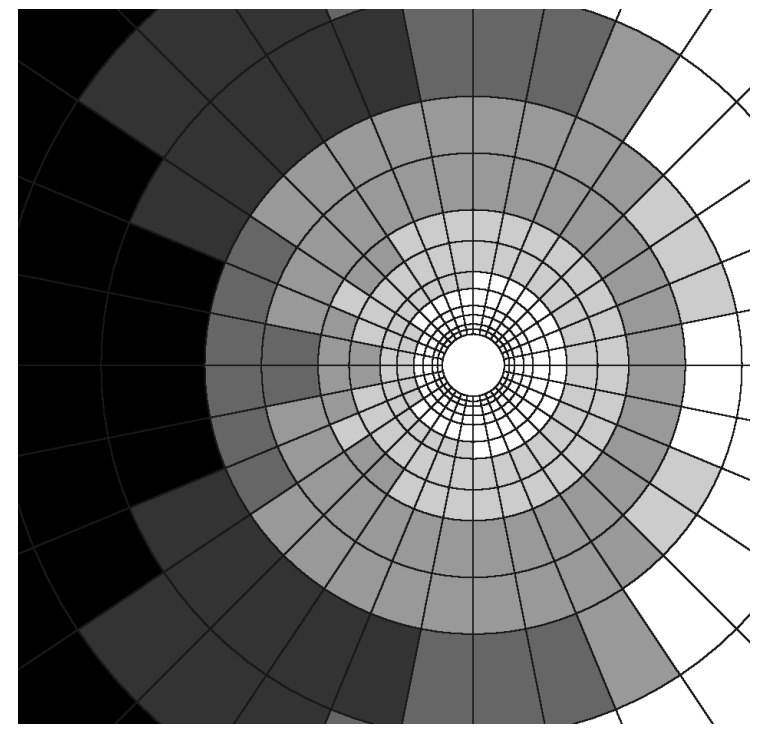

(c) Entropy adjoint

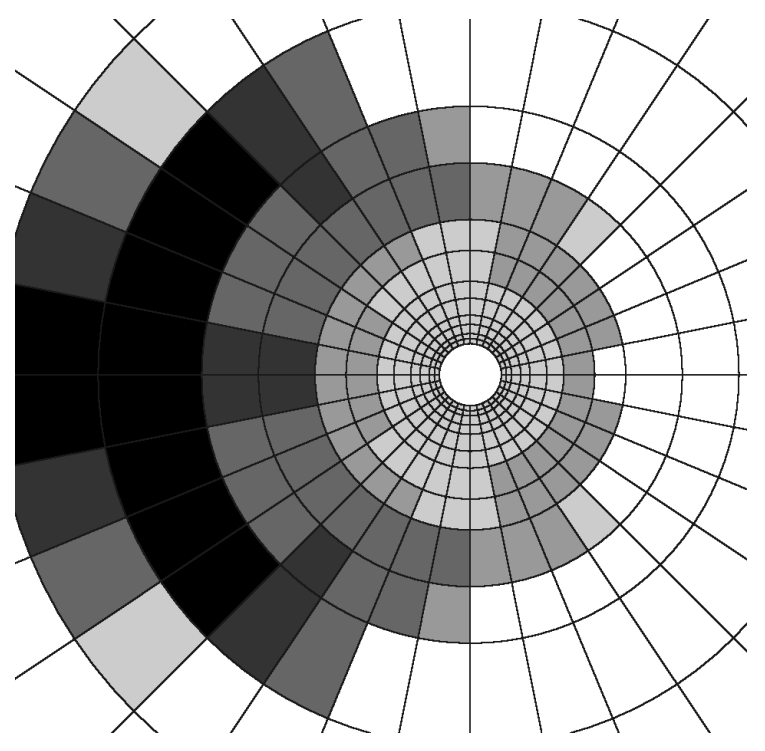

(b) Output adjoint

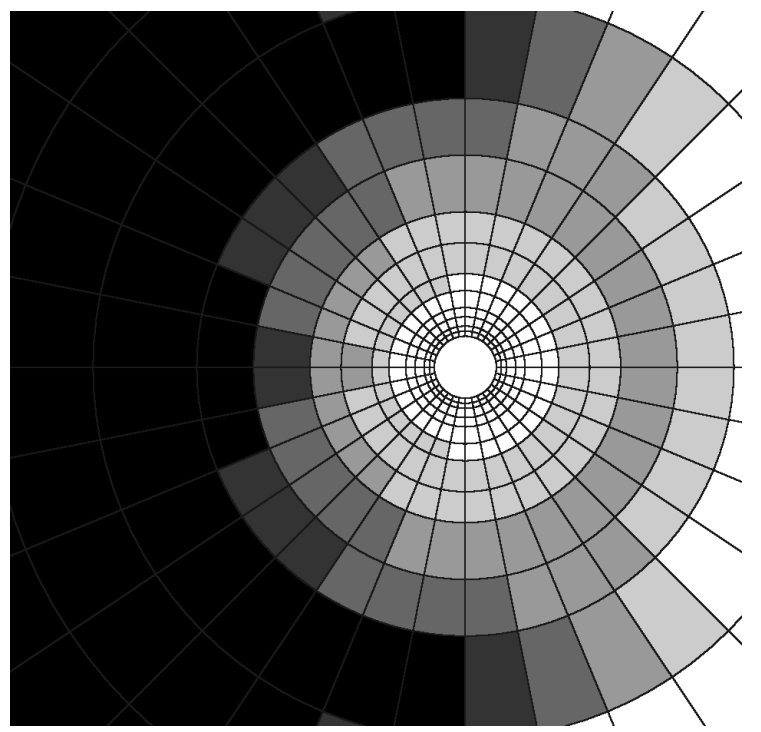

(d) Residual

Figure 13. Two-dimensional depth disturbance: $p$-refinement meshes at the fifth adaptation iteration for various indicators. The gray scale ranges from $p=1$ (white) to $p=6$ (black). 


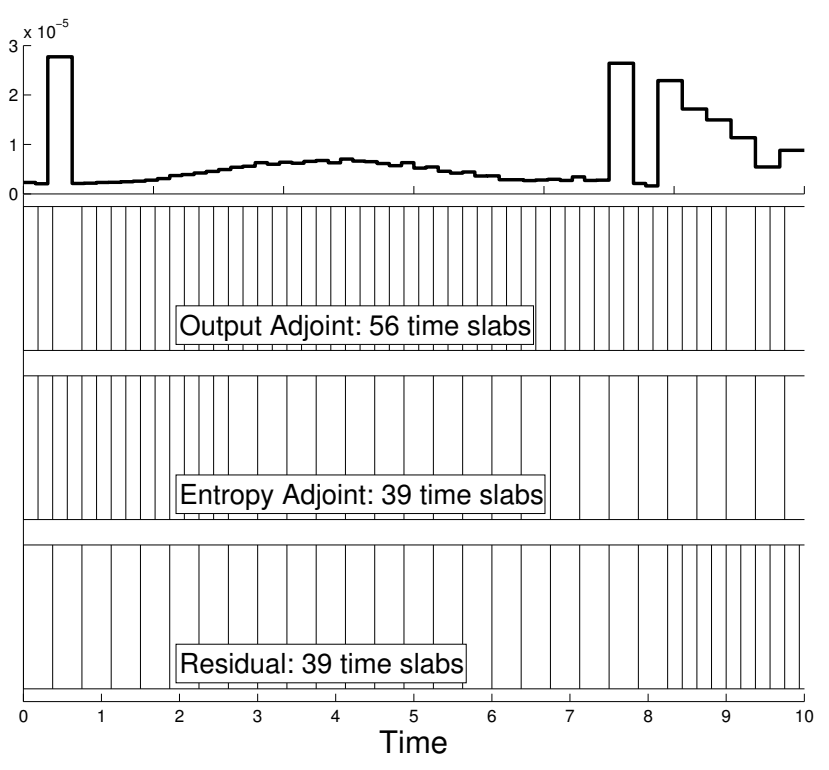

(a) $h$-refinement

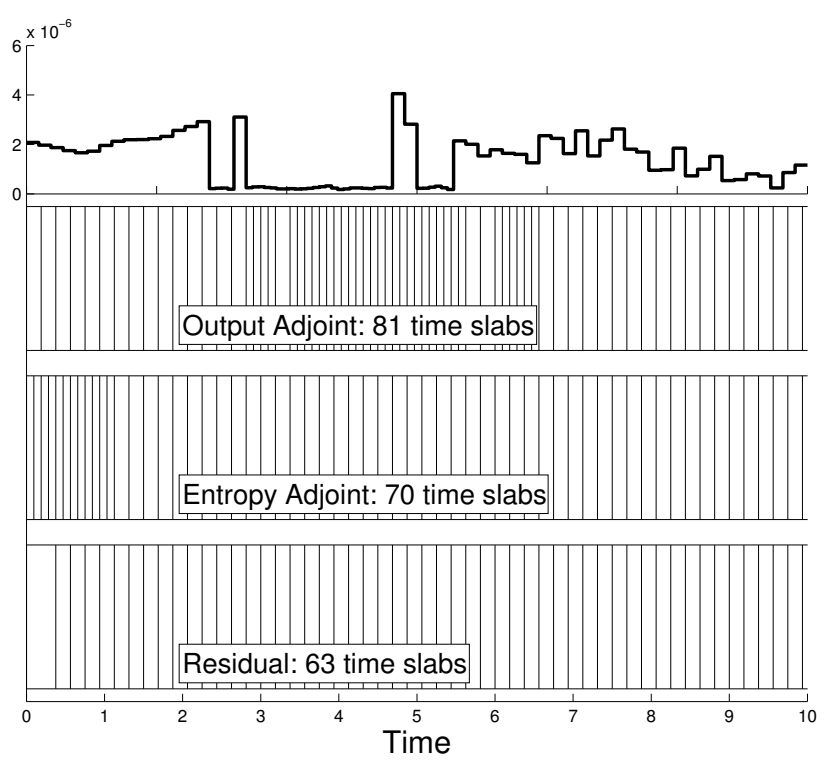

(b) $p$-refinement

Figure 14. Two-dimensional depth disturbance: temporal meshes at the sixth adaptation iteration for various indicators. The top plots represent the error associated with each timeslab for the output adjoint case.

flow problems," AIAA Paper 2007-3944, 2007.

${ }^{13}$ Mani, K. and Mavriplis, D. J., "Error estimation and adaptation for functional outputs in time-dependent flow problems," Journal of Computational Physics, Vol. 229, 2010, pp. 415-440.

${ }^{14}$ Barth, T. J., "Space-time error representation and estimation in Navier-Stokes calculations," Complex Effects in Large Eddy Simulations, edited by S. C. Kassinos, C. A. Langer, G. Iaccarino, and P. Moin, Springer Berlin Heidelberg, Lecture Notes in Computational Science and Engineering Vol 26, 2007, pp. 29-48.

${ }^{15}$ Fidkowski, K. J. and Roe, P. L., "An entropy adjoint approach to mesh refinement," SIAM Journal on Scientific Computing, Vol. 32, No. 3, 2010, pp. 1261-1287.

${ }^{16} \mathrm{Lu}$, J., An a Posteriori Error Control Framework for Adaptive Precision Optimization Using Discontinuous Galerkin Finite Element Method, Ph.D. thesis, Massachusetts Institute of Technology, Cambridge, Massachusetts, 2005.

${ }^{17}$ Hughes, T. J. R., Franca, L. P., and Mallet, M., "A new finite element formulation for computational fluid dynamics: I. Symmetric forms of the compressible Euler and Navier-Stokes equations and the second law of thermodynamics." Computer Methods in Applied Mechanics and Engineering, Vol. 54, 1986, pp. 223-234.

${ }^{18}$ Bova, S. and Carey, G., "An entropy variable formulation and applications for the two-dimensional shallow water equations," International Journal for Numerical Methods in Fluids, Vol. 23, 1996, pp. 29-46.

${ }^{19}$ Richter, T., "Discontinuous Galerkin as time-stepping scheme for the Navier-Stokes equations," Fourth International Conference on High Performance Scientific Computing Modeling, Simulation and Optimization of Complex Processes, Hanoi, Vietnam, 2009.

${ }^{20}$ Anastasiou, K. and Chan, C., "Solution of the 2D shallow water equations using the finite volume method on unstructured triangular meshes," International Journal for Numerical Methods in Fluids, Vol. 24, 1997, pp. 12251245 .

${ }^{21}$ Fidkowski, K. and Luo, Y., "Output-based space-time mesh adaptation for the compressible navier-stokes equations," Journal of Computational Physics, Vol. 230, 2011, pp. 5753-5773.

${ }^{22}$ Roe, P. L., "Approximate Riemann solvers, parameter vectors, and difference schemes," Journal of Computational Physics, Vol. 43, 1981, pp. 357-372.

${ }^{23}$ Bassi, F. and Rebay, S., "GMRES discontinuous Galerkin solution of the compressible Navier-Stokes equations," Discontinuous Galerkin Methods: Theory, Computation and Applications, edited by K. Cockburn and Shu, Springer, Berlin, 2000, pp. 197-208. 